\title{
Christian Gottlob Heyne und die Alte Geschichte
}

\author{
Gustav Adolf LehmanN
}

\section{Vorbemerkung}

Der 2005 verstorbene Göttinger Althistoriker Jochen Bleicken, zweifellos einer der Größten im Fach Alte Geschichte während der 2. Hälfte des 20. Jh.s, hat in einer gründlich dokumentierten wissenschaftsgeschichtlichen Abhandlung - mit dem Titel „Die Herausbildung der Alten Geschichte in Göttingen: Von Heyne bis Busolt" - in seinen Schlussbemerkungen noch einmal eindringlich die fortdauernde Bedeutung Heynes für diese historische und zugleich altertumswissenschaftliche Disziplin hervorgehoben. Bleicken verweist dabei zunächst auf die Pflicht eines jeden Althistorikers, sich im Rahmen seines Faches allen methodischen und sachkritischen Fragestellungen und Herausforderungen aus den modernen historischen Disziplinen zu stellen, um dann fortzufahren: „Doch ist es heilsam für sein Fach, wenn bei allem, was er macht, ihm Christian Gottlob Heyne über die Schulter schaut!“‘2

\section{Zum Status des Fachs Geschichte an der jungen Göttinger Universität}

Das Fach Alte Geschichte bzw. Geschichte des Altertums gab es freilich zu Heynes Zeiten noch nicht - denn als eigenständige akademische Disziplin begegnet die Alte Geschichte unter dieser Bezeichnung erst nach der Mitte des 19. Jh.s; sie ist somit - anders als man bei ihrem (nicht gerade glücklich gewählten) Namen vermuten würde - ein relativ junges Fach. Erwachsen ist diese Disziplin vornehmlich aus zwei unterschiedlichen Wurzeln: Aus einer primär den sog. „Realien“ der Antike zugewandten Richtung der Klassischen Philologie (u. a. in Göttingen) und aus der so genannten „Universalgeschichte“ (u. a. in Leipzig), die sich mit ihrer gesamteuropäischen Perspektive deutlich von der damals

1 Dieser Beitrag basiert in starkem Maße auf der in ihren Hauptkapiteln ungewöhnlich materialreichen und wertvollen Bonner Dissertation von Marianne Heidenreich (Heidenreich 2006). Ein Hinweis auf den Rang dieses grundlegenden Werkes ist hier umso mehr geboten, als in den folgenden Darlegungen natürlich auch Kritik in Einzelheiten begegnen wird.

2 Bleicken 1998, 1033. 
- zumindest in Deutschland - durchgehend dynastisch-territorial orientierten Neueren Geschichte, mit ihren eher partikularen pragmatisch-politischen bzw. staatsrechtlichen oder auch landeskirchlichen Anliegen, abhob. Das primär vom griechisch-römischen Altertum, daneben auch vom Weltbild der Bibel, ausgehende Programm dieser übergreifenden universitären Disziplin ist bekanntlich Gegenstand von Fr. Schillers berühmter Antrittsvorlesung in Jena vom 26. Mai 1789 gewesen: „Was heißt und zu welchem Ende studiert man Universalgeschichte?" Weitaus konkretere Vorstellungen lassen sich jedoch aus den einschlägigen, bereits Jahrzehnte zuvor erschienenen Handbüchern gewinnen, wie sie von Göttinger Historikern, u. a. von Johann Christoph Gatterer (1727-1799) und, besonders wirkungsvoll, von August Ludwig Schlözer (1735-1809) verfasst bzw. redigiert worden sind. ${ }^{3}$

Zur Bedeutung und Rolle des Fachs Geschichte innerhalb der bestehenden Fakultäten und Disziplinen in Göttingen hat sich Heyne schon sehr früh und grundsätzlich (in der universitären Öffentlichkeit) ausgesprochen: Als am 23. Dez. 1766 - mit starker Hilfe aus Hannover in London - ein Institutum Historicum in Göttingen begründet wurde, in dem unter der Leitung Gatterers die wissenschaftliche Arbeit im Bereich der Diplomatik und Heraldik auf eine neue Grundlage gestellt werden sollte, hielt Heyne pflichtgemäß die feierliche lateinische Eröffnungsrede. ${ }^{4}$ Sieht man von dem rhetorischen Überschwang im Genos „Festrede“ einmal ab, so wird deutlich, dass Heyne dem Fach „Geschichte“ hier nur die traditionelle Rolle einer unentbehrlichen Hilfswissenschaft, sowohl für die doctrina sacra der Theologie und die Anliegen der Jurisprudenz als auch für die Interpretationen und Gegenstände der Philologie, zuerkennen wollte, aber durchaus nicht den Rang einer darüber hinaus auch eigenständigen wissenschaftlichen Disziplin.

Selbstverständlich stellt sich an diesem Punkt die Frage, ob Heyne in seinem langen Gelehrtenleben an der hier umrissenen Position stets festgehalten hat, ohne dass sich relevante Modifikationen feststellen ließen. Es gehört m. E. daher zu den Schwächen in der sonst so hilfreichen Studie von Marianne Heidenreich, dass dieser Leitfrage nicht konsequent genug (und nach verschiedenen

3 J. Chr. Gatterer, Handbuch der Universalhistorie (Göttingen 1760, ${ }^{2} 1765$ ); A. L. (v.) Schlözer, Vorstellung seiner Universal-Historie, 1. u. 2. Teil (Göttingen 1772 u. 1773). - S. hierzu auch die Angaben bei Heidenreich 2006 (bes. 149-186) zu den von Heyne selbst, zwischen 1766 und 1772, bearbeiteten und umgeschriebenen Teilbänden einer (ursprünglich in London in 26 Foliobänden erschienenen) Allgemeine(n) Weltgeschichte, s. dazu auch u. S. 68.

4 De studii historici ad omnes disciplinas utilitate, necessitate ac praestantia (Einzeldruck) Göttingen 1766; ferner in Opuscula Academica (collecta et animadversionibus locupletata) tom. I, Göttingen 1785, 280-289. 
Richtungen hin) nachgegangen worden ist. ${ }^{5}$ Im engeren Bereich der Alten Geschichte lässt sich freilich kaum bestreiten, dass es hier für Heyne (ohne jedes „historistische“ Bedenken) zunächst und vor allem um ein großes Reservoir an lehrreichen exempla ging, die sich vorzüglich für die nähere Betrachtung und Einschätzung aktueller Ereignisse und Entwicklungen eigneten. ${ }^{6}$

\section{Heyne und Schlözer: ,Alte' und ,Neue‘ Geschichte}

Der etwas eigenbrötlerische Spezialist Gatterer mochte mit den in Heynes Festrede eröffneten Perspektiven für sein Fach und das Institutum Historicum noch leidlich zufrieden gewesen sein, nicht dagegen der hoch ambitionierte Schlözer, mit dessen Persönlichkeit und universitärer Lehre sich der damals einsetzende Aufschwung einer auch auf staatsrechtlichem und politischem Gebiet profilierten Geschichtswissenschaft in Göttingen eng verbinden sollte. ${ }^{7}$

Tatsächlich lässt sich in der Folgezeit, während der langen Ära der Tätigkeit Heynes in der Georgia Augusta, eine eigentümliche Entwicklung beobachten: Heynes intensive Bemühungen in der Lehre wie auch in wissenschaftlichen Arbeiten, seine Interpretationen und Kommentare zu antiken Autoren und Texten mit dem Rüstzeug solider historisch-philologischer Quellen- und Sachkritik in die jeweiligen Zeitbezüge einzubetten, fanden allmählich immer größeren Anklang in der akademischen Öffentlichkeit. ${ }^{8}$ Gleichzeitig aber zog sich der brillante, freilich auch recht streitbare Schlözer mehr und mehr aus dem Bereich der antiken Geschichte zurück - unter Konzentration auf die publizistisch reizvolleren Aufgaben eines Neuhistorikers und die Thematik der politischen Ver-

5 S. dazu die Darlegungen in der „Einleitung“ von Heidenreich 2006, bes. 18-22 und die sehr verhaltene Bilanz 581-583; vgl. dagegen Schindel 1990, 191-210.

6 Aus Ciceros Katalog der Funktionen und Aufgaben der historia in De orat. II 36 - einem damals in den gebildeten Kreisen Europas allseits bekannten Zeugnis - hat Heyne, wie Heidenreich gezeigt hat (2006, 185f.), eine charakteristische Auswahl getroffen: Ihm ging es - im Sinne der (vor-revolutionären) Aufklärung - vor allem um den (lehrhaften) Gegenwartsbezug, das Potential zur Lenkung und Leitung im privaten wie im öffentlichen Leben (magistra vitae), und eine unbedingte, kritische Wahrheitsliebe (lux veritatis). Als „Trägerin lebendiger Erinnerung“ oder bloß als „Botschafterin längst vergangener Zeiten" (vita memoriae bzw. nuntia vetustatis) war die historia für Heyne von geringerem Interesse.

7 S. U. J. Becher, „August Ludwig von Schlözer“, in: H.-W. Wehler (Hrsg.), Deutsche Historiker Bd. 7 (Göttingen 1980) 7-23; M. Peters, Altes Reich und Europa. Der Historiker, Statistiker und Publizist August Ludwig (v.) Schlözer (1735-1809) (Münster/W. 2003).

8 S. die Angaben bei Heidenreich 2006, 108-112. 
hältnisse und Entwicklungen im zeitgenössischen Staatensystem Europas, deren wissenschaftliche Behandlung damals unter den Begriff „Statistik“ fiel. ${ }^{9}$

Man wird diese Abgrenzungen in der Lehre und (partiell) auch im Schrifttum zwischen Heyne und Schlözer nicht primär auf fachimmanente Spezialisierungen zurückführen dürfen, sondern das besondere Gewicht der Persönlichkeit Heynes und seine außerordentlich breiten, umfassenden Text- und Quellenkenntnisse in Anschlag zu bringen haben. So ist es auch nicht verwunderlich, dass hier in der Folgezeit, unter dem Heyne-Schüler (und Schwiegersohn) Arnold Hermann Heeren (1760-1842), der 1799 die explizit für das Fach „Geschichte" ausgewiesene Professur in Göttingen übernehmen konnte, die Geschichte des Altertums am Ende wieder volles Heimatrecht im Rahmen einer neu belebten und vertieften „Universalgeschichte“ erhalten hat. Im gleichen Jahr (1799) erschien erstmals Heerens großes, immer wieder neu aufgelegtes und noch weit ins 19. Jahrhundert hinein einflussreiches Handbuch der Geschichte der Staaten des Altertums. ${ }^{10}$

\section{Universal- und Altertumsgeschichte im 18. Jahrhundert}

Wenn in diesen Zusammenhängen von „Universalgeschichte“- oder „Allgemeiner Weltgeschichte“ die Rede ist, so handelt es sich freilich - in den äußeren und inneren Dimensionen - um den wie selbstverständlich auf Europa und Vorderasien ausgerichteten Geschichtsraum jener 3000 Jahre, von denen sich damals ein gebildeter Mensch, wie Goethe meinte, „Rechenschaft zu geben“ habe - im klösterlichen Schulunterricht wurde dieser Bereich günstigstenfalls noch ergänzt durch einige Notizen zu dem, was man von der Geschichte und den Dynastien der chinesisch-ostasiatischen Ökumene oder gar von den präkolumbischen Reichen Alt-Amerikas zu wissen glaubte. ${ }^{11}$ All dies aber stellte jedenfalls den Geschichtsraum dar, auf dem das historische Weltbild der Enzyklopädisten und

9 Seine bedeutendste, weit in den Bereich der Politik ausgreifende Unternehmung stellte die Gründung und Herausgabe der Zeitschriften Schlözers Briefwechsel meist historischen und politischen Inhalts (1778-1782) und anschließend der Staatsanzeigen (1782-1793/4) dar.

10 Handbuch der Geschichte der Staaten des Altertums, mit besonderer Rücksicht auf ihre Verfassung, ihren Handel und ihre Colonien (zuerst 2 Bände, die fünfte Auflage des umfangreichen Werkes erschien 1828 in 6 Bänden). - Zu der Originalität und dem methodischen Rang dieses Handbuches siehe auch Bleicken 1998, 1010-1012.

11 Ein instruktives Beispiel stellt hierfür der Lehrstoff des - während der 1720er und 1730er Jahre - in den von Jesuiten betriebenen Gymnasien (collegia) in Böhmen verbreiteten Geschichtslehrbuchs dar: Rudimenta Historica, bes. opusc. IV: De regnis, aliisque Orbis Provinciis. 
Aufklärer um die Mitte des 18. Jahrhunderts, aber auch noch die Geschichtsphilosophie von Georg Wilhelm Hegel und die seines berühmtesten Schülers, des „Links-Hegelianers“ Karl Marx, basierten.

Im Rahmen der üblichen „Universalgeschichte“ wurde damals in handbuchmäßigem Überblick stets auch über die antike Geschichte Ägyptens und Mesopotamiens - nach den griechisch-römischen Quellen - gehandelt. Heynes Besprechungen in den Göttingischen Anzeigen (hierzu von durchgehend größerem Umfang) lassen ebenso wie die Notizen zur Allgemeinen Weltgeschichte erkennen, dass er für diese Bereiche in der Geschichte des Altertums (jenseits der griechisch-römischen Antike im engeren Sinne), aber auch für die „Dunklen Zeiten" der Frühgeschichte von Hellas und Italien ein ausgeprägtes Interesse besaß. ${ }^{12}$ Gleichwohl ist klar, dass sich in Heynes Vorstellungen vom Gang der Alten Geschichte keinerlei Vorahnung von jener ungeheueren Erweiterung der historischen Perspektiven findet, die sich für die antiken Hochkulturen Ägyptens und Vorderasiens fortschreitend ergeben sollten - mit der philologischen Entschlüsselung der Originalquellen und der archäologischen Erschließung der historischen Schauplätze seit der Mitte des 19. Jh.

Handelt es sich doch um den Hinzutritt von nicht weniger als zwei mit dramatischem politischen und soziokulturellen Leben hoch angefüllten Jahrtausenden in unsere eigene, europäisch - mediterrane Kulturwelt hinein. Diese geradezu revolutionäre Ausweitung unserer Vorstellungen von den Epochen der Geschichte des Altertums, zu der schließlich auch die Entdeckung der kretischminoischen und der mykenisch-frühgriechischen Hochkulturwelt gehört, ist primär den sich erst im späten 19. und frühen 20. Jahrhundert voll entfaltenden Disziplinen der Ägyptologie und Altorientalistik mitsamt den jeweils zugehörigen Archäologien zu verdanken. ${ }^{13}$ Aus dieser eigentlich banalen Feststellung ergibt sich allerdings auch, dass man die erwähnten geschichtsphilosophischen Systeme von Hegel ebenso wie von Marx und Engels, die noch ganz der „Uni-

12 Seit 1770 war Heine - neben seinen Verpflichtungen als Professor der Klassischen Philologie sowie als professor eloquentiae und als Leiter der Universitätsbibliothek - zugleich zum „perpetuierlichen“ Secretär der „Königlichen Gesellschaft der Wissenschaften“ eingesetzt (vgl. dazu auch Nesselrath, u. S. 171 und mit der Herausgabe der bereits 1739 gegründeten Göttinger Rezensionszeitschrift (GGA) beauftragt worden (bis zu seinem Tode 1812). Aus seiner Feder stammen mehr als 6200 (zum größeren Teil allerdings nur kurze) Buchbesprechungen.

13 Auf dieser Basis konnte dann Eduard Meyer (1855-1930) - gewissermaßen in zwei groBen Anläufen (mit der ersten Auflage ab 1884 u. der zweiten ab 1909) - daran gehen, seine Konzeption einer umfassenden Geschichte des Altertums (für die mediterrane Welt und ganz Vorderasien) auf der Grundlage gleichmäßiger Quellenkenntnis zu verwirklichen. 
versalgeschichte“ alten Schlages verpflichtet waren, weder in ihrer Methodik noch in ihren substanziellen Aussagen überschätzen darf.

\section{Heynes eigene Arbeiten zur Universal- und Altertumsgeschichte}

Heyne ist - mit der o. begründeten, grundsätzlichen Einschränkung - in seinen stets nüchternen, quellenbezogenen Arbeiten jedenfalls den zeitgenössischen Anforderungen einer sowohl altertumswissenschaftlichen als auch ,universalhistorischen" Betrachtungsweise in hohem Maße gerecht geworden: In den mannigfachen Korrekturen und Ergänzungen, die er zu einer neuen, kritischen Ausgabe (in deutscher Sprache) der (von William Guthrie u. John Gray edierten) General History of the World beisteuerte (zwischen 1765-1772), hat er sich, wie Ulrich Schindel gezeigt hat, als überaus kenntnisreicher „Universalhistoriker“ erwiesen - gerade auch jenseits der Grenzen des griechisch-römischen Altertums. ${ }^{14}$ Das gründlich überarbeitete Werk trug den vertrauten Titel „Allgemeine Weltgeschichte“. Heynes eindringliche Kritik an den Autoren des originalen englischen Sammelwerkes - dass sie nämlich durchgehend zu wenig Sorgfalt und Konsequenz in der Quellenkritik und bei der Auswahl unter den antiken Autoren zeigten -, ist in jeder Hinsicht bemerkenswert und kann m. E. vielfach auch noch heute als aktuell gelten. ${ }^{15}$

Auf die großen Leistungen Heynes, der die seit Jahrhunderten vorwiegend unter rein antiquarischen Aspekten behandelten „Realien“ des Altertums funktional, innerhalb ihrer konkreten Lebenswirklichkeit, zu interpretieren suchte, wird in mehreren Beiträgen dieses Bandes eingegangen. In der Behandlung archäologisch-kunsthistorischer Themen, in der Numismatik und sogar in der Etruskologie hat er für die akademische Lehre und in der damaligen Forschungsdiskussion ganz neue Wege beschritten. Man wird ihn daher ohne Einschränkungen als einen der Gründerväter der modernen, interdisziplinären Altertumswissenschaft anzusehen haben. Insofern ist auch die Frage durchaus berechtigt, ob es überhaupt sinnvoll sein kann, die Gestalt dieses großen, vielseitigen Gelehrten noch weiteren Rubrizierungen zuzuordnen?

Eine Antwort lässt sich hier wohl am ehesten mit einem Blick auf eine besondere Gruppe von Studien und Erörterungen geben, die in höherem Maße als die GGA-Rezensionen Heynes Leistungen gerade im Bereich der Alten Geschichte

14 S. Schindel 1990, bes. 201f.

15 Als einschlägiges Beispiel könnte man heute $u$. a. an den spielerisch-eleganten, aber auch leichtfertigen Umgang mit der Alexander-Überlieferung in dem international weit verbreiteten Buch des Althistorikers Robin Lane Fox erinnern: Alexander the Great (London 1973; deutsche Ausg. Düsseldorf 1974). 
und der in ihr beschlossenen politischen Entwicklungen dokumentieren: Es geht um einige specimina aus Heynes Universitätsprogrammen, deren Erstellung zu den Amtspflichten ihres Autors als professor eloquentiae an der Georgia Augusta gehörte. Zum größeren Teil handelt es sich dabei freilich nur um relativ kurze, lateinisch abgefasste Schriften, mit denen mehrmals im Jahr - gewissermaßen als „Beilagen“ (unter der Bezeichnung prolusiones) - zu einer akademischen Feier eingeladen wurde. Zunächst gab es in Göttingen drei feststehende Termine im Jahr, zu denen an alle Universitätsangehörigen sowie auch an anwesende Honoratioren Einladungen zu versenden waren: vor allem der jeweils halbjährige Wechsel im Amt des Prorectors in der Leitung der Universität (am 2. Januar und 2. Juli) - denn rector magnificentissimus der Georgia Augusta war damals der englische König und amtierende Kurfürst von Hannover höchstselbst (und dies ganz ohne zeitliche Begrenzungen). Hinzu kam noch das Stiftungsfest der Universität am 17. September. ${ }^{16}$ Heyne hat diese kleinformatigen, aber stets mit einem starken pädagogischen, gelegentlich auch politischen Impetus verfassten Gelegenheitsschriften, die sich an die Studentenschaft und ein breites akademisches Publikum richteten, offenkundig sehr geschätzt: Regelmäßig erschienen überarbeitete Versionen oder ausführliche Referate über diese Arbeiten in deutscher Sprache in den Göttingischen Anzeigen, und zwar stets an prominenter Stelle in den Stückfolgen. Darüber hinaus wurden von ihm die lateinischen Originaltexte mit umfangreichen Ergänzungen und Anmerkungen seit 1785, in insgesamt sechs Bänden, noch einmal als Opuscula Academica collecta et animadversionibus locupletata publiziert - im Sprachstil des gängigen akademischen Gelehrten-Lateins.

Im letzten Band dieser Opuscula Academica von 1812, dem Todesjahr Heynes, firmiert der Autor - neben seinem Göttinger Professorentitel - freilich nicht mehr als „Hofrat“ bzw. als „Geheimer Justizrat“ der königlich-britannischen Majestät, sondern in Anpassung an die schwierigen Zeitläufte als „Ritter des Ordens der Westphälischen Krone“ (comes coronae westphalicae). Dieser Band, der nur noch zu einem Teil Programmschriften des bekannten Typus enthält, ist zugleich ein beeindruckendes Dokument der harten politischen Zäsuren von 1806/7 im nordwestdeutschen Raum und lässt deutlich die nach Innen und AuBen prekäre Lage der Georgia Augusta unter der Herrschaft des Königs Jérôme Bonaparte in Kassel erkennen.

$16 \mathrm{Ab} 1784$ wurde mit ähnlichem Aufwand auch der Geburtstag des regierenden Königs Georg III. (1760-1820) alljährlich (am 4. 6.) gefeiert. Erst in den Kriegs- und Unruhejahren ab 1792/93 wurden diese jährlichen Festfeiern und damit auch die Zahl der fälligen Programm-Schriften auf zwei reduziert - bis mit der französischen Besetzung Göttingens (1805) diese akademischen Festbräuche längerfristig ganz suspendiert wurden. 


\section{Alte Geschichte und Zeitgeschichte in Heynes prolusiones}

Manche dieser insgesamt 126 prolusiones / Abhandlungen beschäftigen sich im engeren Sinne mit philologischen, autorenbezogenen Fragestellungen, andere enthalten allgemeine Betrachtungen zur Moral und zum antiken Gesellschaftsund Kulturleben; darüber hinaus finden sich in ihnen auch aufschlussreiche Anmerkungen zum damaligen Wissenschaftsbetrieb. Für uns aber sind die zahlreichen Schriftstücke von Bedeutung, die - oftmals durch politische Ereignisse und Entwicklungen aus Heynes Gegenwart angeregt - sich mit Themen und exempla aus der historischen Welt des Altertums befassen. Den Bedenken, die sich in methodischer Hinsicht gegen derartige Versuche, politische Erfahrungen der Zeitgeschichte unmittelbar mit Episoden der antiken Welt zu verknüpfen, erheben, steht als Gewinn eine bis dahin ungewohnte Lebendigkeit in den um Realitätsnähe bemühten Skizzierungen gegenüber. Der später von B. G. Niebuhr (1776-1831) erhobenen Forderung, dass es in der antiken Geschichte wesentlich darauf ankomme, sich ,philologische Überlieferung als wirklich“ vorzustellen, ist Heyne hier wiederholt sehr nahe gekommen.

In den Zeiten des noch unangefochtenen Ancien Régime, also bis 1790, ist allerdings, wie sich aus der grundlegenden Arbeit von Marianne Heidenreich ergibt, der sachliche Zusammenhang zwischen dem aktuellen Anlass und dem Inhalt der althistorischen Abhandlung oft nicht allzu eng: Ein gutes Beispiel dafür bietet das Zusammentreffen der Zarin Katharina II. und des Kaisers Joseph II. auf der Krim (vor dem Hintergrund des zweiten russisch-türkischen Krieges) und den von Heyne - mit ausdrücklichem Bezug zu diesen aktuellen Ereignissen - erarbeiteten Programmschriften. ${ }^{17}$

Tatsächlich treten in diesen prolusiones die politischen Positionen und Überzeugungen des „Aufklärers“ Heyne ebenso offen zu Tage wie seine historischen Vorstellungen von den großen Epochen der Geschichte des Altertums. Dies gilt besonders für sein leidenschaftlich-positives Interesse an der Griechischen Geschichte: Denn die wichtigste Aufgabe bzw. das Grundprinzip des Staates sieht der Göttinger Professor in der Sicherung der persönlichen und allgemeinen Freiheit als Grundbedingung für ein moralisch gutes und glückliches Leben der Menschen.

Diese Position teilte Heyne bekanntlich mit den meisten zeitgenössischen Repräsentanten der vorrevolutionären „Aufklärung“ in Europa ebenso wie das

17 „Rerum Chersonesi Tauricae memoria breviter exposita“ (zum 2.7.1787, vgl. GGA 1787, S. 1257-1259) sowie Opuscula Academica, tom. III (1788) S. 384-397 und ein Jahr später: „Longinquarum in barbaros expeditionum, et barbarorum in Europam incursionis, consilia nostris aetatibus pariter sublata" (zum 2.7.1788, vgl. GGA 1788, S. 1217-1220) sowie Opuscula Academica, tom. IV (1796), S. 32-48. 
Interesse an den großen Gesetzgebern der Griechen wie Lykurgos, Zaleukos oder Solon, die in dieser Hinsicht schlechthin Vorbildliches geleistet hätten. Mit der Freiheit als Grundwert aber waren für Heyne auch das Ideal arbeitsamer Frugalität und eine grundsätzliche Ablehnung von Luxus und Verschwendung eng verbunden. $\mathrm{Zu}$ den forschungsgeschichtlich bedeutsamen Anliegen, die Heyne im Rahmen dieser Abhandlungen vorgetragen hat, zählen u. a. seine Bemühungen um eine angemessene Berücksichtigung der Geschichte der ,West-Griechen" (in Unteritalien und Sizilien), vor allem aber auch sein reges Interesse an der Literatur- und Kulturgeschichte des Zeitalters, das wir heute - in Anlehnung an die (keineswegs einheitlichen) Vorstellungen J. G. Droysens (1808-1884) - als Epoche des „Hellenismus“ bezeichnen. ${ }^{18}$

Sein durchaus kritisches Bild vom Verlauf der Römischen Geschichte basiert dagegen weitgehend auf den für ihn generell wichtigen Hauptwerken Montesquieus (den Considérations sur les causes de la grandeur des Romains et de leur décadence, 1734, und De l'esprit des lois, 1748): Dabei geht es vorrangig um die These, dass die Römische Republik gerade durch ihre großen kriegerischen Erfolge, die sich daraus ergebende machtpolitische Expansion und eine entsprechende soziale Auflösung im Innern die Grundlagen ihrer republikanischen WerteOrdnung selbst zerstört habe, so dass sie am Ende zwangsläufig einer Despotie anheim fallen musste.

Auf spezielle historische Probleme und exempla aus der Römischen Geschichte kommt Heyne in seinen prolusiones daher auch erst im Rahmen seiner intensiven Auseinandersetzung mit der Französischen Revolution zu sprechen. Hierbei spielte eine gewisse Rolle wohl auch die von den Radikalen in Paris so gern praktizierte Maskerade mit Gestalten, Themen und Attitüden aus der antiken Tradition über die besonders „,tugendhafte“, frühe Römische Republik.

Anfänglich hatte Heyne freilich, wie die meisten Repräsentanten der „Aufklärung" in Deutschland, die revolutionäre Bewegung in Frankreich lebhaft begrüßt und die schon in der Frühphase der Bewegung erkennbare Neigung zu grausamen Gewaltakten zunächst noch als Spätfolge der in seinen Augen in jeder Hinsicht desaströsen Politik des „Sonnenkönigs“ Ludwig XIV. verstehen wollen. Aber auch später hat Heyne, der Schwiegervater des berühmten Naturforschers, JakobinerFreundes und aktiven Mainzer Revolutionärs Georg Forster (1754-1794), ohne Abstriche an seinen freiheitlichen (letztlich republikanischen) Grundüberzeugungen festgehalten: Als sich 1794 die Anzeichen mehrten, dass von Hannover und

18 S. hierzu grundsätzlich die Studie von R. Kassel, Die Abgrenzung des Hellenismus in der griechischen Literaturgeschichte (Berlin / New York 1987 = ders., Kleine Schriften, hrsg. v. H.-G. Nesselrath [Berlin / New York 1991] 154-173) bes. auch 11 (=165f.) mit Anm. 25 zu Heynes Abhandlung „De genio saeculi Ptolemaeorum“, 1763 (= Opuscula Academica I 76-85). 
London aus in die Lehr- und Publikationsfreiheit an der Georgia Augusta eingegriffen werden sollte, hat Heyne in einer prolusio - mit breiten Exkursen in die antike Geschichte - auf die Sinnlosigkeit und offenkundige Schädlichkeit solcher Interventionen in ungewöhnlich leidenschaftlichem Ton hingewiesen. ${ }^{19}$ Danach hat Heyne allerdings die Themen seiner Programmschriften weitgehend ,entpolitisiert" und auf diese „Kurskorrektur“ auch ausdrücklich im Referat zu einer Programmschrift vom Sommer 1796 hingewiesen..$^{20}$ Nach Heynes Bekunden sollte seine in den prolusiones nun über eine Reihe von Jahren der antiken Kunstgeschichte und einigen lateinischen Autoren der Spätantike zugewandte Thematik auch als persönlicher Dankbarkeitserweis für die zeitweilige politische Beruhigung in seiner Lebenswelt verstanden werden. Denn durch den preußisch-französischen Sonderfrieden von Basel (im April 1795) war inzwischen ganz Norddeutschland (bis zur Mainlinie) zu einer von beiden Mächten garantierten Neutralitätszone bestimmt worden. Auch der englische König war alsbald - freilich nur für sein Kurfürstentum Hannover - diesem Sonderfrieden beigetreten, der sich nicht zuletzt auf die Entfaltung der Weimarer Klassik (in dem wichtigen Jahrzehnt zwischen 1795-1805) wohltätig auswirken sollte.

Deutlich hebt sich die gründliche Auseinandersetzung Heynes mit der Französischen Revolution von der zuvor gezeigten, eher verhaltenen Reaktion auf den Umbruch in Amerika (seit 1776) und den Verlauf des Unabhängigkeitskrieges der 13 nordamerikanischen Staaten gegen Großbritannien ab: In seinen prolusiones hat er sich hier mit deutlichen Hinweisen und Urteilen erst positioniert, als sich bereits der Sieg der Insurgenten abzeichnete, und dann nach Bezugspunkten für exempla aus dem Altertum Ausschau gehalten: Für Heyne ist es primär die Weisheit der antiken Metropolen (,Mutterstädte“) - bei den Hellenen wie auch seitens der Römer - in der großzügigen und fürsorglichen Behandlung ihrer Tochterstädte bzw. coloniae, die sich überaus positiv von der in der Gegenwart von der englischen Führung praktizierten Politik abgehoben habe.

Damit wird nachdrücklich auf die starre Haltung des Königs und den gescheiterten Herrschaftsanspruch des britischen Parlaments gegenüber den Amerikanern verwiesen, deren Mehrheit zunächst nur nach angemessener Selbstverwaltung und Mitbestimmung verlangt habe. Nach dem Friedensschluss von 1783 hält Heyne im übrigen für die Regierung in London dann auch bald schon den historisch-

19 Diese Programmschrift trägt den Titel: „Litterarum bonarum studia, tamquam imperiis infesta, perperam proscripta“" (zum 1. März 1794, GGA 1794, 393-397 unter dem Titel: „Sind die Wissenschaften der Ruhe des Staats verderblich, und verdienen sie ausgerottet zu werden?"; s. ferner Opuscula Academica IV, 1796, 416-442; zu weiteren Publikationen dieser kämpferischen Protestschrift s. die Nachweise bei Heidenreich, 2006, 603).

20 GGA 1796, 1610 (zu der Programmschrift: „Philostrati imaginum illustratio II“ vom 1.9.1796); vgl. ferner die praefatio zum 5. Bd. der Opuscula Academica, Göttingen 1802, p. IV f. 
politischen „Trost“ bereit, dass nach Ausweis der zur Verfügung stehenden antiken exempla eine Staaten-Konföderation - wie die der ehemaligen amerikanischen Kolonien - wohl kaum über längere Zeit politischen Bestand haben werde. ${ }^{21}$

\section{Heyne über Phokion und römische Agrargesetzgebung}

Zum Abschluss unseres knapp bemessenen Überblicks sei noch kurz auf zwei für die weitere Entwicklung einer wissenschaftlich-kritischen Althistorie bedeutsame Studien in Heynes prolusiones-Schriften hingewiesen: Es handelt sich zum einen um die 1787 vorgelegte, den bisher üblichen Umfang deutlich überschreitende Untersuchung zur Politik und Gestalt des athenischen Strategen und Politikers Phokion, der nicht nur der von Demosthenes und anderen athenischen Politikern vertretenen Außenpolitik Athens (gegen den Aufstieg Makedoniens im Zeitalter Philipps II. und Alexanders d. Gr.), sondern auch der Polis-Demokratie seiner Zeit mit äußerster Skepsis gegenüberstand. ${ }^{22}$ In dieser Abhandlung hat Heyne, wie nahezu ein Jahrhundert später der große Philologe Jakob Bernays (1824-1881) ausdrücklich festgestellt hat, ${ }^{23}$ in seiner nüchtern-kritischen Destruktion des traditionellen, im 18. Jh. in Frankreich wie in Deutschland weit verbreiteten Idealbildes von Phokion eine überzeugende und im Kern noch immer gültige quellenkritische Leistung erbracht: In der vita Plutarchs war Phokion, der persönlich auch in engen Beziehungen zu Platons Akademie gestanden hatte, auf sehr einseitiger Quellenbasis zu einer vorbildlich-loyalen, in ihrer Tragik an Sokrates heranreichenden Heldenfigur hochstilisiert worden.

Heyne beruft sich demgegenüber zu Recht auf andere, weniger panegyrische Überlieferungen bei Diodor und Nepos. Auch wenn Heyne seine Befunde nicht zu einer tiefer gehenden Kritik der Plutarch-Viten ausgeweitet hat, traf seine Studie, wie nicht zuletzt die heftige Reaktion Schlözers zeigt, durchaus einen Nerv in den damals gängigen historisch-politischen Vorstellungen. ${ }^{24}$

21 „De belli Romanorum socialis caussis et eventu, respectu ad bellum cum Coloniis Americanis gestum habito“, vom 2.7.1783; GGA 1783 S. 1193-11195, Opuscula Academ. III, 1788, 144-161. S. ferner „Foederatarum rerump. coalitio vix umquam satis fida, exemplis ex antiquitate illustrata“, vom 18.9.1783; GGA 1783, 1627-1631; Opuscula Academ. III, 1788, 162-183. Weitere Hinweise bei Heidenreich 2006, 196.

22 „Res a Phocione in rep. Atheniensium gestae in disceptationem vocatae“, zum 2.1.1787; GGA 1787, 81-86; Opuscula Academ. III, 1788, 344-364. Zur erregten zeitgenössischen Diskussion über die von Heyne ermittelten Befunde s. auch Heidenreich 2006, 19.

23 J. Bernays, Phokion und seine neueren Beurtheiler. Ein Beitrag zur Geschichte der griechischen Philosophie und Politik (Berlin 1881).

24 Als Beispiel, wie sehr Phokion zeitweilig als politisches Idol im späten 18. Jh. (in Frankreich wie in Deutschland) gegolten hat, kann wohl auch die Tatsache gelten, dass sich in Goethes Haus am Frauenplan der Gipsabguss von der römischen Kopie eines atheni- 
Die zweite Studie, auf die hier noch kurz hingewiesen werden soll, ist die Programmschrift „Leges agrariae, pestiferae et execrabiles“ von 1793, in der Heyne vehement gegen die historischen Prätentionen und aktuellen Forderungen der „Agrairions“-Bewegung in Paris Stellung bezogen hat. ${ }^{25}$ Deren Agitation für eine umfassende, bis an die Schwelle zum Kommunismus heranführende Loi Agraire endete bekanntlich erst mit der Katastrophe ihres Anführers „C. Gracchus“ Babeuf und seines Geheimbundes ,der Gleichen“ im Frühjahr 1797.

Heyne kommentiert in seiner Schrift mit großem Eifer alle überlieferten politischen Anläufe in Rom zu leges agrariae-Reformen - von der frühen Römischen Republik bis zur Ära der Triumvirn nach Caesars Ermordung - und trifft dabei quellen- wie sachkritisch genau den Punkt im römischen Bodenrecht, der für seinen jüngeren Zeitgenossen B. G. Niebuhr zum Ausgangspunkt einer neuen, weitaus kritischeren Analyse der gesamten römischen Überlieferung über die frühe Republik geworden ist. Und Niebuhr hat hier die Abhängigkeit von seinem akademischen Lehrer Heyne immerhin auch klar bekannt. ${ }^{26}$

Warum also ist nicht Heyne, der kenntnisreiche und inspirierende Lehrer, sondern Niebuhr (und daneben auch Heeren) - weit über den deutschsprachigen Kreis hinaus - zu dem Ruf gelangt, die Geschichte des Altertums als eine wissenschaftlich-kritische, historische Disziplin begründet zu haben? Auf diese Frage lässt sich wohl eine einfache Antwort geben: Heyne war und blieb bis an sein Lebensende - als Hochschullehrer und Seminardirektor, als professor eloquentiae, und höchst erfolgreicher Bibliotheksdirektor, ferner als ,,perpetuierlicher“ Secretär der Gesellschaft der Wissenschaften und Redaktor der GGA - in allzu viele akademische Aufgabenbereiche eingebunden. So fehlten ihm schlechterdings Zeit und Kraft, um neben der Philologie seine vertieften Kenntnisse von der Geschichte und den Kulturen des Altertums, vor allem aber seine Befähigung zu strenger, umfassender Quellenkritik an einer größeren historischen Darstellung zu erproben. Gemessen an Heerens Handbuch (s. o.) und Niebuhrs Römischer Geschichte (von 1811/12 in 2 Bänden) blieben die prolusiones in ihrer großen Zahl und der Vielfalt ihrer Themen - allen Bemühungen ihres Autors zum Trotz - am Ende doch nur rasch konsumierte Gelegenheitsschriften.

schen Strategen-Bildnisses befindet, das damals ausdrücklich auf Phokion bezogen worden ist.

25 Siehe A. Heuss, Barthold Georg Niebuhrs wissenschaftliche Anfänge. Untersuchungen und Mitteilungen über die Kopenhagener Manuskripte und zur europäischen Tradition der lex agraria (loi agraire) (Göttingen 1981) passim.

26 Siehe dazu jetzt die Studie von W. Nippel, „Barthold Georg Niebuhr und die Begründung der modernen Althistorie“", in: A. M. Baertschi / C. G. King (Hrgg.), Die modernen Väter der Antike. Die Entwicklung der Altertumswissenschaft an Akademie und Universität im Berlin des 19. Jahrhunderts (Berlin / New York 2009) [8-113] 102. 